\title{
PREPARATION AND CHARACTERIZATION OF A HYBRID SOLID POLYMER ELECTROLYTE CONSISTING OF POLY(ETHYLENEOXIDE) AND POLY(ACRYLONITRILE) FOR POLYMER-BATTERY APPLICATION
}

\author{
Munichandraiah Nookala \\ Department of Inorganic and Physical Chemistry \\ Indian Institute of Science, Bangalore 560012 (India) \\ Phone : 91-80-309-2240 FAX : 91-80-334-1683 \\ Lawrence G. Scanlon and Richard A. Marsh \\ Wright Laboratory/Battery Electrochemistry Section \\ Wright-Patterson AFB, OH 45433-7251 (U.S.A.) \\ Phone : 937-255-7770 FAX : 937-656-7259
}

\begin{abstract}
For application in an ambient temperature solid state lithium battery, a highly dimensionally-stable polymer electrolyte based on polyethyleneoxide (PEO) suffers from low ionic conductivity, whereas a highly conducting gel electrolyte based on polyacrylonitrile (PAN) suffers from low dimensional stability. In order to overcome these problems, a hybrid solid polymer electrolyte (HSPE) was prepared using PEO, PAN, propylene carbonate (PC), ethylene carbonate (EC) and lithium perchlorate. The HSPE films were highly conducting as well as dry, freestanding and dimensionally-stable. The films were characterized by constructing symmetrical cells containing non-blocking lithium electrodes and also blocking stainless steel electrodes. Investigations were carried out on ionic conductivity, electrochemical reaction, interfacial stability and morphology of the films. The properties of HSPE were compared with the films prepared using (i) $\mathrm{PEO}$ and $\mathrm{LiClO}_{4}$, and (ii) PAN, PC, EC and $\mathrm{LiClO}_{4}$. The results suggest that the HSPE is a potential electrolyte material for application in a polymerbattery.
\end{abstract}

\section{INTRODUCTION}

In recent years, research activities for the development of high energy and environmental-friendly rechargeable battery systems are at the forefront of efforts in the field of electrochemical power sources. Rechargeable lithium batteries occupy the top most importance among this category. There is an urgent need of these batteries for applications such as battery powered electric vehicles. Additionally, experimental programmes for newer applications, e.g., aerospace applications are recently reported [1]. Rechargeable solid state lithium batteries employing a solid polymer electrolyte (SPE) is expected to yield specific energy as high as $150-200 \mathrm{Wh} \mathrm{kg}^{-1}$ with a good cycle life. These batteries are yet to be realized in the commercial market. In addition to contributing for enhancement of specific energy of the battery, the SPE provides further more advantages, viz., (i) SPE itself functions as the inter electrode separator, thus eliminating the need of a conventional battery separator, (ii) reactivity of lithium by corrosion is expected to be less in a solid medium, and (iii) the anodic dissolution and cathodic deposition of lithium from SPE is expected to be uniform, which avoids the formation of dendrites.

For the purpose of using in a battery, the SPE should primarily possess a high ionic conductivity (specific conductivity, $\sigma \geq 10^{-3} \mathrm{~S} \mathrm{~cm}^{-1}$ ) at ambient temperature. This level of $\sigma$ appears to be an essential requirement for achieving performance of the battery comparable to the performance of a liquid electrolyte - based lithium battery. In addition to possessing high ionic conductivity, the SPE films must satisfy a number of important chemical, electrochemical and mechanical criteria[2]: (i) the SPE films must have an electrochemical stability domain up to about $4.5 \mathrm{~V}$; (ii) they must be chemically comapatible 
with lithium anode and the cathode; (iii) they need to possess good thermal stability; (iv) they must be processable as free-standing films; (v) they need to possess good dimensional stability under electrode stack pressure in a cell; (vi) the SPE films should be capable of withstanding stresses caused by morphological changes which the electrodes undergo during cycling; and (viii) they also need to possess good mechanical strength. However, either inadequate ionic conductivity or poor dimensional stability of the SPE film is one of the serious problems, as briefly described below, hampering the development of this battery system.

Extensive studies have been reported on polyethyleneoxide (PEO) as a SPE medium. Specific conductivity $(\sigma)$ is of the order of $10^{-8} \mathrm{~S} \mathrm{~cm}^{-1}$ at ambient temperature, when a SPE film is prepared by complexing PEO with a lithium salt. This value is very low and not suitable for battery applications, although the SPE film has good dimensional stability. Several procedures for preparation of highly conducting $\left(\sigma \geq 10^{-3} \mathrm{~S} \mathrm{~cm}^{-1}\right) \mathrm{SPE}$ films are reported in the literature. One of the procedures is to immobilize a solution of propylene carbonate (PC) and ethylene carbonate (EC) containing a lithium salt (e.g., $\mathrm{LiClO}_{4}$ ) in a host polymer[3]. Immobilization of the solution imparts a high specific conductivity $\left(\sigma \geq 10^{-4} \mathrm{~S}\right.$ $\mathrm{cm}^{-1}$ ) to the host polymer, viz,, polyacrylonitrile (PAN). Even though a high specific conductance is achieved with PAN electrolytes, dimensional stability of these films is poor. They are gels rather than solid films. Inadequate dimensional stability is due to the existence of a liquid phase entrapped in PAN matrix. Unlike PEO which is a solvent for lithium salts, PAN does not seem to solvate the salts to a significant extent. Indeed, it merely encapsulates the solution in its matrix and the ionic conduction takes place in the liquid medium.

In the present studies, solid polymer electrolyte films consisting of PEO, PAN, $\mathrm{PC}, \mathrm{EC}$ and $\mathrm{LiClO}_{4}$ were prepared to overcome the above described problems inherent to PEO and PAN electrolyte films. The results suggest that these hybrid solid polymer electrolyte (HSPE) films possess good mechanical strength as well as high ionic conductivity. Several characterization studies are reported.

\section{EXPERIMENTAL}

Polyethyleneoxide (MW : $4 \times 10^{6}$ ), polyacrylonitrile and ethylene carbonate were used as received from Aldrich. Propylene carbonate (Aldrich) was distilled under reduced pressure and stored over molecular sieves of grade $4 \mathrm{~A}$ before use. For preparation of HSPE films, appropriate quantities of $\mathrm{PEO}, \mathrm{PC}, \mathrm{EC}$ and $\mathrm{LiClO}_{4}$ were first dissolved in acetonitrile. The required quantity of PAN was added and the solution was stirred for several hours to ensure uniform dispersion of the undissolved PAN. A film was cast by spreading the suspension on a Teflon covered glass plate and allowing acetonitrile to evaporate slowly. Finally, the film was dried at $80^{\circ} \mathrm{C}$ under vacuum for about $10 \mathrm{~h}$. The resulting HSPE film was visually examined for its dry and free-standing nature.

For the purpose of comparison, (i) films consisting of PEO and $\mathrm{LiClO}_{4}$ and (ii) gel films of PAN, PC, EC and $\mathrm{LiClO}_{4}$ were also prepared. While the former SPE film was prepared by solution casting technique, the gel film was prepared by mixing the required quantities of the constituents thoroughly and heating in a glass petri dish at $90{ }^{\circ} \mathrm{C}$ for about $5 \mathrm{~min}$. On cooling, a transparent gel film was obtained.

Symmetrical cells of the type : SS/SPE/SS and (SS)Li/SPE/Li(SS) were assembled in an argon filled dry box. SS refers to a stainless steel disc with an integral lead. The SS disc was in contact with SPE as a blocking electrode in the former type of cells. It was contacting $\mathrm{Li}$ electrode in the latter type of cells. The non-blocking $\mathrm{Li}$ electrode of $1 \mathrm{~cm}^{2}$ area was punched from $0.75 \mathrm{~mm}$ thick Li ribbon supplied by Aldrich. The cells were assembled in Teflon holders and were contained in air-tight glass containers which had provision for taking electrical contacts. The glass container of the cell was heated to a defined temperature by using a heating tape and a temperature controller. The cell was kept to equilibrate at the set temperature, maintained within $\pm 1{ }^{\circ} \mathrm{C}$, for about 2 $\mathrm{h}$ prior to the experiment. Experiments were carried out at different temperatures between ambient temperature and $100{ }^{\circ} \mathrm{C}$.

Electrochemical impedance spectroscopic studies of the symmetrical cells were performed in the frequency range between $100 \mathrm{mHz}$ and $100 \mathrm{kHz}$ using Electrochemical Impedance Analyzer PARC(EG\&G) Model 6310 . The analysis of the data was carried out using the computer programme based on non-linear least square fitting procedure written by Bouckamp and supplied by PARC (EG\&G). Samples of SPE films were examined under Cambridge Instuments Scanning Electron Microscope model S-360.

\section{RESULTS AND DISCUSSION}

(i) Electrochemical Impedance Spectroscopy: 
The impedance spectra of (SS)Li/SPE/Li(SS) symmetrical cells containing SPE as (i) $\mathrm{PEO}_{8} \mathrm{LiClO}_{4}$, (ii) PAN gel and (iii) HSPE films are shown in Fig. 1 as Nyquist plots. The spectrum in Fig. la contains two apparent semi circles, whereas the spectra in Figs. $1 \mathrm{~b}$ and 1c contain a single semi circle each. All semi circles, however, are distorted. The high frequency semi circle close to the origin in Fig. la is due to the response of parallel combination of the polymer film resistance $\left(R_{b}\right)$ and geometric capacitance $\left(\mathrm{C}_{\mathrm{g}}\right)$. The diameter of the semi circle provides the value of $R_{b}$. The other semi circle at low frequency domain in Fig. 1a represents the properties of Li/SPE interface. Contributions from the chargetransfer reaction as well as from the passivating film present on lithium are taken into account. If the two time constants due to the parallel $R_{c t}$ and $C_{d l}$; and parallel $R_{f}$ and $C_{f}$ are well separated, then two semi circles corresponding to these two processes are expected to be found clearly. However, the high distortion of the semi circle suggests that the two time constants are similar in magnitude. Therefore, it is assumed that the distorted semi circle is due to overlap of two semi circles, one corresponding to the charge-transfer process (i.e., $R_{\text {ot }}$ and $\mathrm{C}_{\mathrm{dl}}$ ) and the other corresponding to the passivating film on lithium metal (i.e., $R_{f}$ and $C_{f}$ ).

The impedance spectra (Fig. $1 \mathrm{~b}$ and 1c) of symmetrical cells having PAN gel and HSPE as electrolyte films do not show the high frquency semi circle in contrast to the case of $\mathrm{PEO}_{8} \mathrm{LiClO}_{4}$ film. As the resistances $\left(R_{b}\right)$ of the former films are very low, the low values of the time constants $\left(=R_{b} C_{g}\right)$ do not fall into the experimental frequency range. As discussed above, the distorted semi circle is treated for the interfacial phenomenon comprising electrochemical reaction and passivating film on lithium metal.

\section{(ii) Evaluation of Resistances:}

It is clear from the above discussion that the electrochemical impedance data are associated with several resistances and capacitances. The intention of the present investigations being the evaluations of values of resistances, simple equivalent circuit models are employed. As the centers of the involved semi circles of the Nyquist plots appear to fall below the real axis, capacitances are substitued by constant phase elements following the studies reported in the literature[4]. Equivalent circuits employed in present investigations are shown in Fig. 2. The equivalent circuit shown in Fig. $2 a$ is used to evaluate the resistances from the data of Fig.la. The circuit shown in Fig. $2 b$ is used to evaluate the resistances from the data of Figs. 1b and 1c. A typical fit- plot of experimental data are shown in Fig. 3. A good agreement between the measured data and theoretical data suggest that the procedure followed in analysis of the experimental results is reliable.

The SS/HSPE/SS symmetrical cells were employed for obtaining conductance of HSPE films by recording electrochemical impedance spectra. The Nyquist plot of the spectrum contained the experimental data points linearly dispersed instead of a semi circle. Intercept of the linear plot at the real axis was taken as the resistance of the HSPE film.

(iii) Ionic conductivity:

The specific conductivity $(\sigma)$ at the SPE was calculated using the following equation :

$$
\sigma=1 / R_{b} A
$$

where 1 is the thickness of the electrolyte film and A its cross-sectional area ( $=$ area of the electrode)

The aim of the investigations was to arrive at an appropriate composition of HSPE which possessed a high specific conductivity as well as dry with good dimensional stability. The HSPE consists of several components, viz., PEO, PAN, PC, EC and $\mathrm{LiClO}_{4}$, and therefore experiments required for arriving at an appropriate composition, taking account of all components and their contributions, are rather large in number. As the enhancement in conductivity is due to the presence of PC and EC, several HSPE films were prepared only by varying the concentration of $\mathrm{PC}$ and $\mathrm{EC}$ while maintaining concentrations of other components nearly invariant. The conductivity $(\sigma)$ of the HSPE films at 30 ${ }^{\circ} \mathrm{C}$ as a function of $\left[(\mathrm{PC}+\mathrm{EC}) / \mathrm{LiClO}_{4}\right] \mathrm{mol}$ ratio is shown in Fig. 4. Also, $\sigma$ values of HSPE films of several mole ratios are shown in Fig. 5 as a function of temperature. It is evident that the HSPE film having 3.67 mole ratio of $\left[(\mathrm{PC}+\mathrm{EC}) / \mathrm{LiClO}{ }_{4}\right]$ exhibits the highest conductivity at all temperatures. The weight ratio of PEO:PAN:PC:EC: $\mathrm{LiClO}_{4}$ in this HSPE film, thus, becomes $1: 0.75: 0.75: 0.45$. The specific conductivity, $\sigma$ at $30{ }^{\circ} \mathrm{C}$ is $3.7 \times 10^{-4} \mathrm{~S} \mathrm{~cm}^{-1}$. This value is about four orders of magnitude higher than $\sigma=1 \times 10^{-8} \mathrm{~S} \mathrm{~cm}$ of $\mathrm{PEO}_{8} \mathrm{LiClO}_{4}$. However, it is lower by one order of magnitude in comparision with $\sigma=1.34 \times 10^{-3} \mathrm{~S} \mathrm{~cm}^{-1}$ of gel electrolyte film made of $\mathrm{PAN}, \mathrm{PC}, \mathrm{EC}$ and $\mathrm{LiClO}_{4}$. The advantage, at the cost of slightly decreased $\sigma$, is that the HSPE film is dry with good mechanical strength.

The temperature dependence of of HSPE film is found to follow Arrhenius relation as shown in Fig. 6. This behaviour of HSPE film is in contrast to the 
behaviour of $\mathrm{PEO}_{8} \mathrm{LiClO}_{4}$ films, which show a transition at about $70{ }^{\circ} \mathrm{C}$ due to melting of PEO. In the case of $\mathrm{PEO}_{8} \mathrm{LiClO}_{4}$ films, thus, there are two temperature regions. In the region below about $70^{\circ} \mathrm{C}$, the temperature coefficient is larger in relation to the region above $70^{\circ} \mathrm{C}$. Altough PEO is present as one of the components of HSPE, the effect of its meiting is not present in the Arrhenius plot. The activation energy of conduction of HSPE obtained from Fig. 6 is $33.8 \mathrm{~kJ} \mathrm{~mol}^{-1}$. Similar values of activation energy are reported for PAN-based electrolytes.

\section{(iv) Electrochemical Reaction and Passive Film on Lithium:}

The charge-transfer resistance $\left(R_{c t}\right)$ represents the kinetics of the electrochemical reaction between lithium metal and $\mathrm{Li}^{+}$ion in the solid polymer electrolyte film:

$$
\mathrm{Li}^{+}+\mathrm{e}^{-}=\mathrm{Li}
$$

The exchange current density $\left(\mathrm{I}_{0}\right)$ of the reaction (2) was evaluated as

$$
\mathrm{I}_{0}=\mathrm{RT} / \mathrm{FAR}_{\mathrm{ct}}
$$

Although eqn. (3) is generally applied to a reaction occurring at a film-free mertal, it is also used frequently to evaluate reaction (2) occurring at $\mathrm{Li}$ metal which is covered with an inherent passive film. Reaction (2) proceeds through the passive film present between the $\mathrm{Li}$ metal and the electrolyte film.

The values of exchange current density $\left(I_{0}\right)$ of reaction (2) are 1.3, 3.7 and $0.64 \mu \mathrm{A} \mathrm{cm}$ in HSPE, PAN gel and $\mathrm{PEO}_{8} \mathrm{LiClO}_{4}$ electrolyte films respectively at 30 ${ }^{\circ} \mathrm{C}$. Although the value of $\mathrm{I}_{\mathrm{o}}$ is slightly lower in HSPE than in PAN gel, it is higher than the value in $\mathrm{PEO}_{8} \mathrm{LiClO}_{1}$. Exchange current values about three orders of magnitude higher than that in HSPE were reported for reaction (2) in similar highly conducting gel electrolytes [5]. These studies, however, were made with freshly deposited lithium on a nickel substrate. The lower values of $I_{o}$ obtained in the present studies can be attributed to a surface film present on lithium. The cyclic voltammograms recorded with the (SS)Li/SPE/Li(SS) symmetrical cells did not contain current peaks. Instead, steady state type of voltammograms with limiting currents were obtained. This suggests that the electron-transfer rate is slower than the rate of diffusion of $\mathrm{Li}^{+}$ion at $\mathrm{Li} / \mathrm{SPE}$ interface. The reason for not obtaining current peaks is probably due to highly resistive passive film which was already formed on $\mathrm{Li}$ before recording voltammograms.

From the resistance $R_{f}$, the thickness of passive film on lithium was calculated using eqn. (3). The specific conductivity of the passive film was taken as $1 \times 10^{-9} \mathrm{~S} \mathrm{~cm}^{-1}$ from literature[6]. The thickness of passive film on lithium in freshly assembled cell containg HSPE is obtained as $40 \AA$.

The complex plane impedance plots at different intervals of ageing of (SS)Li/HSPE/Li(SS) cells are shown in Fig. 7. It is seen that both the high and low frequency intercepts on real axis keep shifting towards higher values. The cells were aged for about $2000 \mathrm{~h}$ at ambient temperature, resistance values were evaluated at several intervals of ageing. It was found that resistance of HSPE $\left(R_{b}\right)$, charge-transfer resistance $\left(R_{c t}\right)$ and thickness of passive film on Li surface (t) gradually increase during ageing of the cells. When lithium metal is in contact with the electrolyte film, it tends to undergo oxidation (or, corrosion) and the reaction products accumulate resulting in the formation of a passive film on $\mathrm{Li}$. As the ageing time progresses, the passive film grows in thickness. Since reaction (2) has to proceed through the passive film, it experiences a higher resistance $\left(R_{c t}\right)$ with increasing ageing time. Accordingly, exchange current density of the reaction decreases. Similar type of increase in $R_{\mathrm{ct}}$ of reaction (2) in solid polymer electrolyte media is reported in literature.

The decrease in $\sigma$ is also attributed to the corrosion of $\mathrm{Li}$ metal. The electrolyte film contains liquid components viz., PC and EC. It is known that $\mathrm{Li}$ is thermodynamically unstable when in contact with $\mathrm{PC}$ or EC electrolytes. The liquid solvent molecules undergo reductive decomposition which in turn facilitates the oxidation of Li metal. The decrease in $\sigma$ on ageing may be due to loss of PC and EC molecules in the electrolyte film. This process leads to changes in composition of the electrolyte film with respect to liquid phase and overall $\mathrm{Li}^{+}$ion concentration in the composite medium. The concentration of $\mathrm{Li}^{+}$ions can also be considered changing due to partial and slow dissolution of outer layers of passive film into the electrolyte medium.

\section{(v) Scanning Electron Microscopy:}

SEM micrograph of HSPE is shown in Fig. 8. Long interlocked chains of PAN are clearly found. The liquid components viz., $\mathrm{PC}$ and $\mathrm{EC}$ containing $\mathrm{LiClO}_{4}$ together with PEO are probably encapsulated within the interlocked network. This type of structure is perhaps responsible for retaining the liquid components within the film and keeping the surface of the film dry.

\section{CONCLUSIONS}

The hybrid polymer electrolyte films studied in the present investigations exhibit ionic conductivities in 
the range of $10^{-4} \mathrm{~S} \mathrm{~cm}^{-1}$ at ambient temperature. This value is much higher than the conductivity of conventional PEO-based polymer electrolyte films $(\sigma \approx$ $\left.10^{-8} \mathrm{~S} \mathrm{~cm}^{-1}\right)$. Even though there is a marginal decrease in $\sigma$ in comparison with PAN gel films, the advantage achieved with HSPE film is that it is dry and free-standing with dimensional stability as good as the PEO polymer electrolyte film.

\section{ACKNOWLEDGEMENTS}

Financial support of this work by U.S. Air Force through their European Office of Aerospace Research and Development (U.K.) under Contract No. C0007 is gratefully acknowledged.

\section{REFERENCES}

1.Chmielewski, A. B., Surampudi, S., Bennet, B., Frank, H., and Mueller, R., 1995, "Lithium Battery Space Experiment", Proceedings of the 30th IECEC, Vol. 1, Yogi Swami, D., Kannberg,L. D., Mancini, T. R., and S.
Somasundaram, eds., The American Soc. of Mechanical Enginneers, NY., pp. 67-72.

2. Alamgir, M., and Abraham, K. M., 1994, "Room Temperature Polymer Electrolytes", Lithium Batteries, G. Pistoia ed., Elsevier, Amsterdam, pp.93-134.

3. Alamgir, M., and Abraham, K. M., 1990, J. Electrochem. Soc., Vol. 137, p. 1657.

4. Wu, X., Zhang, W., and Yu, H., 1995, J. Electroanal. Chem., Vol. 398, p.1.

5. Christie, A. M., and Vincent, C. A., 1996, J. Appl. Electrochem., Vol. 26, p.255.

6. Montesperelli, G., Nunziante, P., Pasquali, M.,and Pistoia, G., 1990, Solid State Ionics, Vol. 37, p. 149.
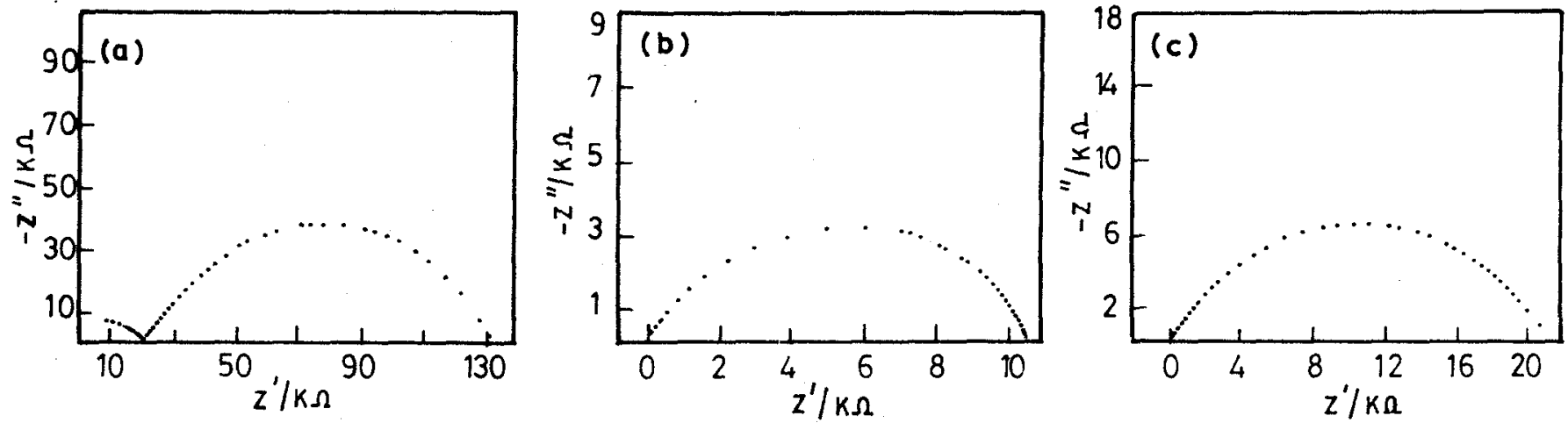

Figure 1. Nyquist plots of (SS)Li/SPE/Li(SS) cells at ambient temperature. The SPE is (a) $\mathrm{PEO}_{8} \mathrm{LiClO}_{4}$, (b) PAN gel and (c) HSPE.

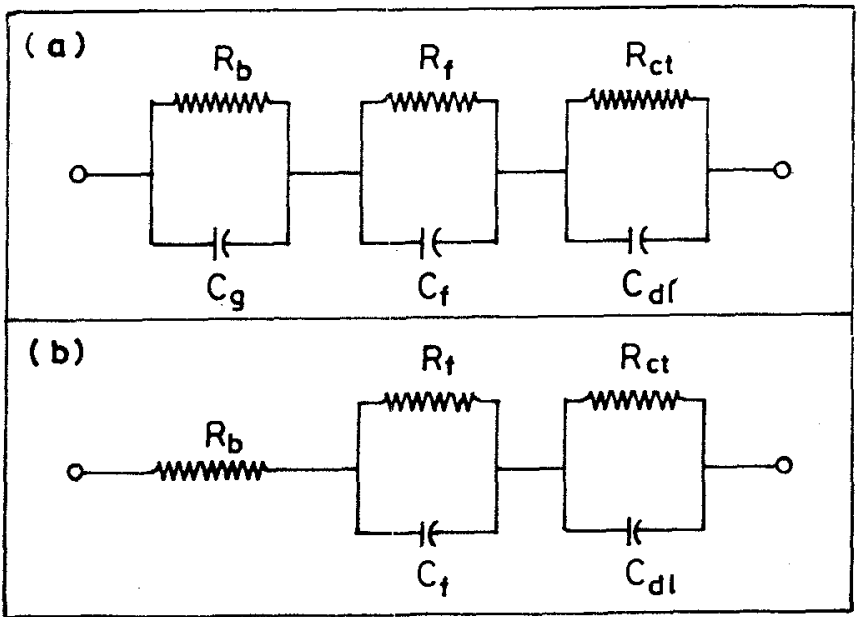

Figure 2. Equivalent circuits employed for fitting the experimental data. 


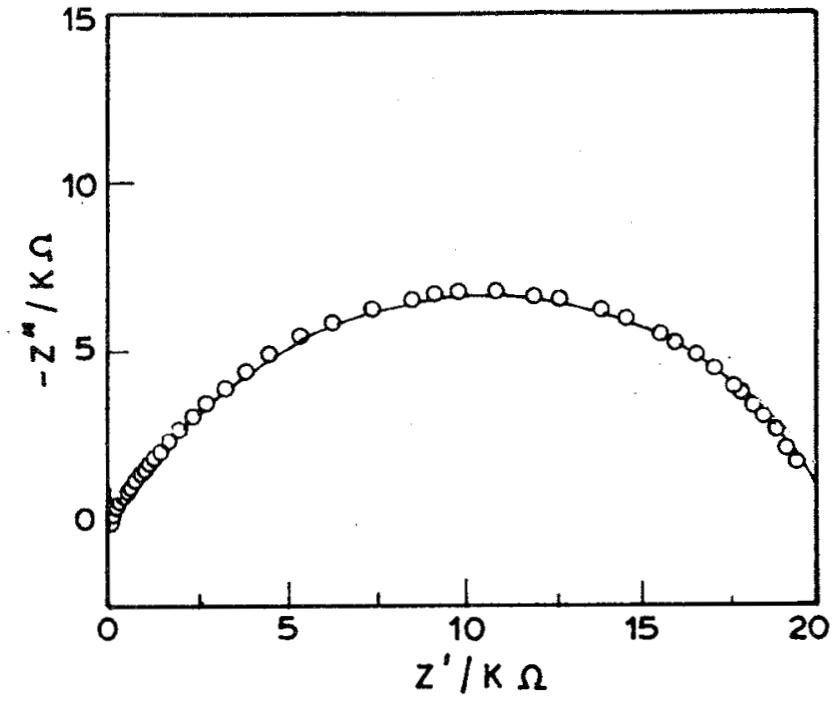

Figure 3. Nyquist plot of (SS)Li/HSPE/Li(SS) cell at ambient temperature with experimental (--) and theoretical (000) data.

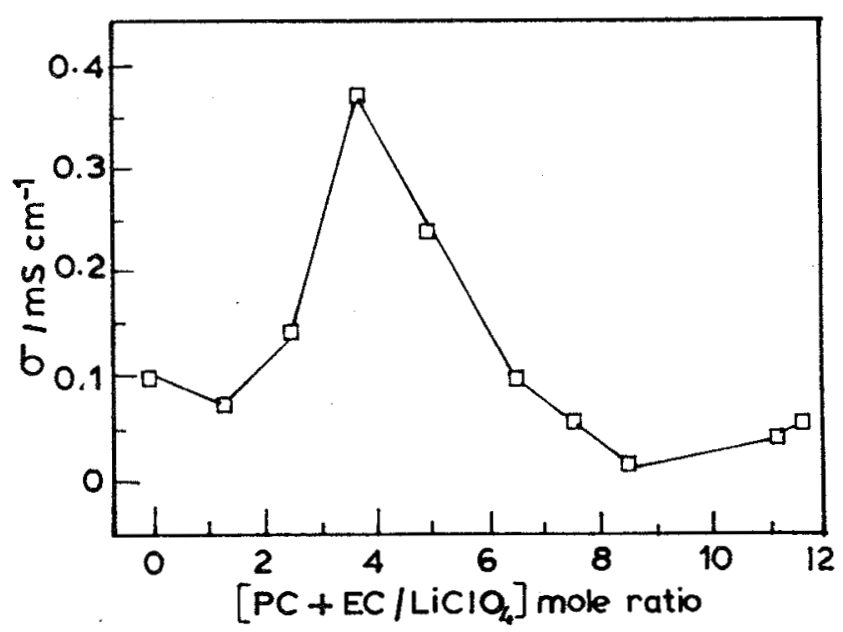

Figure 4. Specific conductivity $(\sigma)$ at $30{ }^{\circ} \mathrm{C}$ against $\left[\mathrm{PC}+\mathrm{EC} / \mathrm{LiClO}_{4}\right]$ mole ratio in HSPE film.

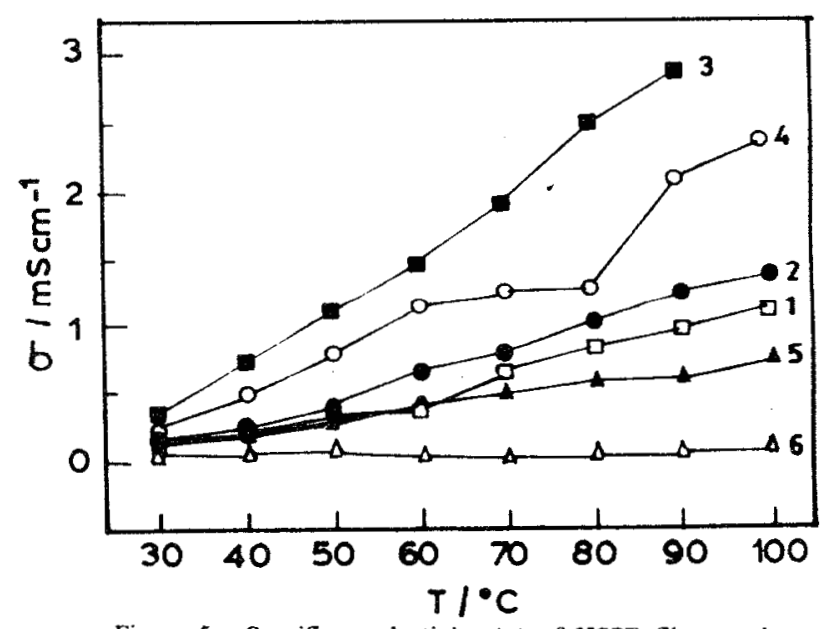

Figure 5. Specific condudivity $(\sigma)$ of HSPE films against temperature at $\left[\mathrm{PC}+\mathrm{EC} / \mathrm{LiClO}_{4}\right]$ mole ratio(1) 0 , (2) 2.5, (3) 3.75 , (4) 5.0 , (5) 6.5 and (6) 8.5 .

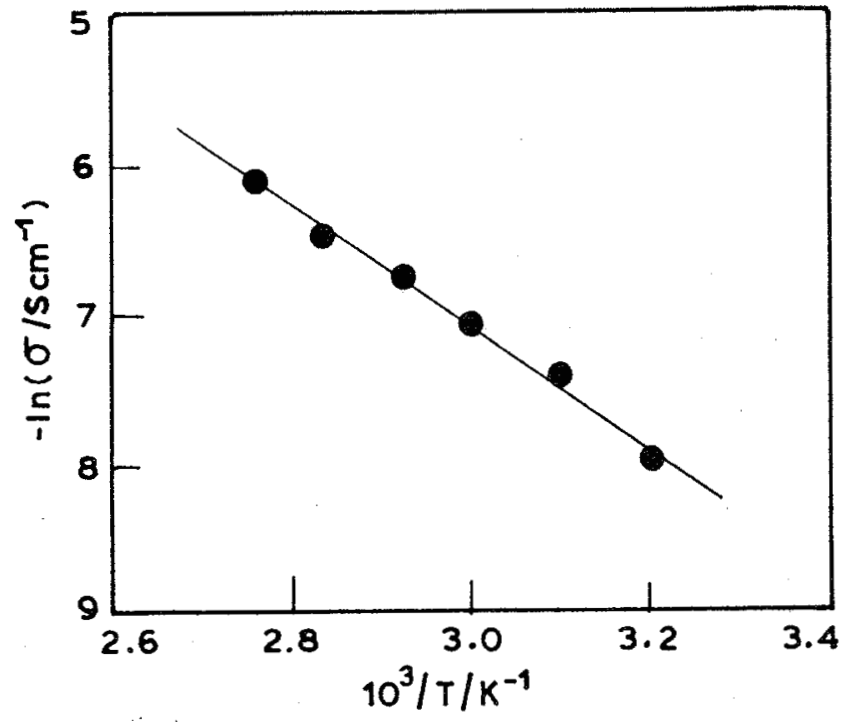

Figure 6. Arrhenius plots of specific conductivity $(\sigma)$ of HSPE film.

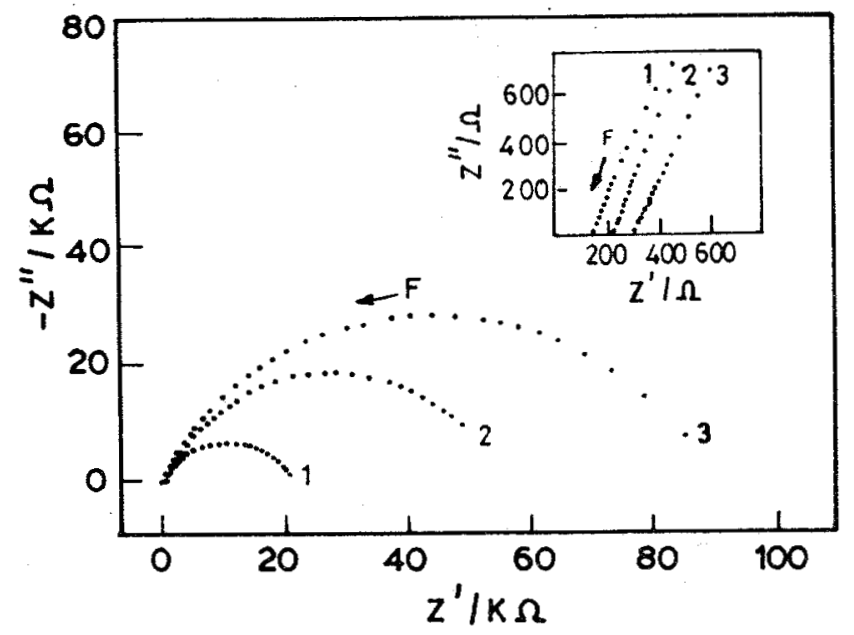

Figure 7. Nyquist plots of (SS)Li/HSPE/Li(SS) cell aged for (1) $48 \mathrm{~h}$, (2) $144 \mathrm{~h}$, and (3) $312 \mathrm{~h}$ at ambient temperature. The high frequency intercept is expanded and shown as inset.

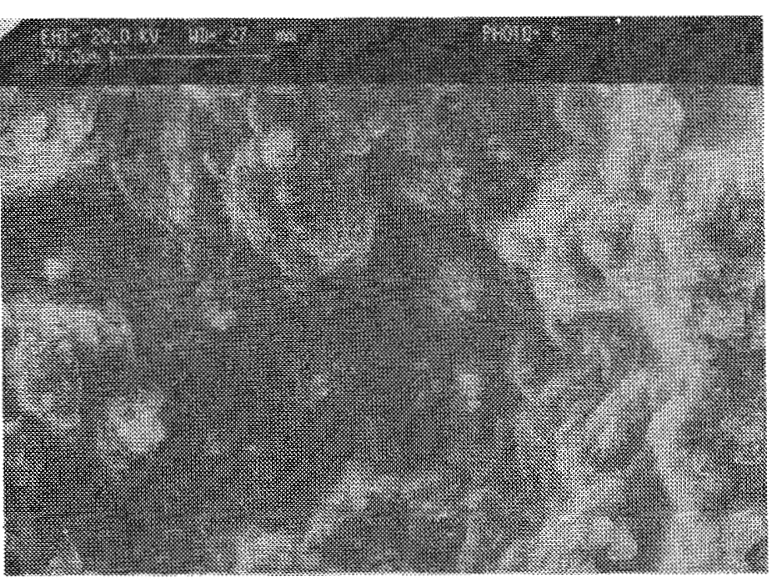

Figure 8. SEM micrograph of HSPE film. 